\title{
Using high-throughput sequencing of ITS2 to describe Symbiodinium metacommunities in St. John, U.S. Virgin Islands
}

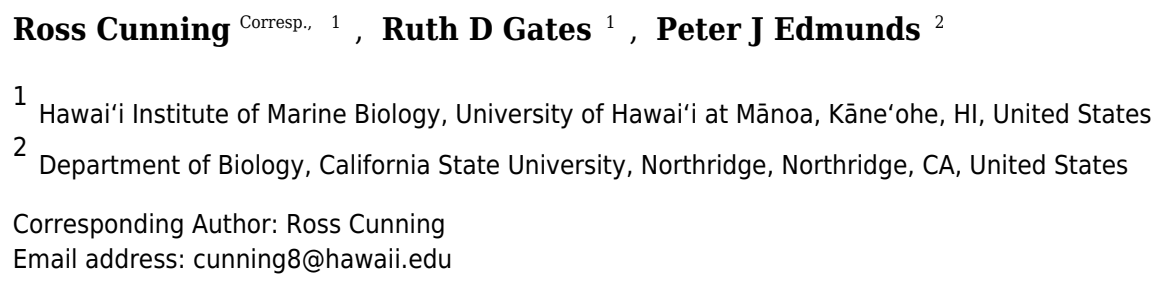

Symbiotic microalgae (Symbiodinium spp.) strongly influence the performance and stresstolerance of their coral hosts, making the analysis of Symbiodinium communities in corals (and metacommunities on reefs) advantageous for many aspects of coral reef research. High-throughput sequencing of ITS2 nrDNA offers unprecedented scale in describing these communities, yet high intragenomic variability at this locus complicates the resolution of biologically meaningful diversity. Here, we demonstrate that generating operational taxonomic units by clustering ITS2 sequences at $97 \%$ similarity within, but not across, samples collapses sequence diversity that is more likely to be intragenomic, while preserving diversity that is more likely interspecific. We utilize this 'within-sample clustering' to analyze Symbiodinium from ten host taxa on shallow reefs on the north and south shores of St. John, US Virgin Islands. While Symbiodinium communities did not differ between shores, metacommunity network analysis of host-symbiont associations revealed Symbiodinium lineages occupying 'dominant' and 'background' niches, and coral hosts that are more 'flexible' or 'specific' in their associations with Symbiodinium. These methods shed new light on important questions in coral symbiosis ecology, and demonstrate how application-specific bioinformatic pipelines can improve the analysis of metabarcoding data in microbial metacommunity studies. 
1 Using high-throughput sequencing of ITS2 to describe Symbiodinium metacommunities in St.

2 John, U.S. Virgin Islands

3 Ross Cunning ${ }^{*}$, Ruth D. Gates ${ }^{1}$, Peter J. Edmunds ${ }^{2}$

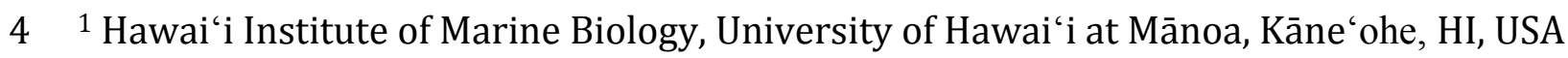

$5 \quad 2$ Department of Biology, California State University, Northridge, CA, USA

6 * Corresponding author: ross.cunning@gmail.com

7

8

9

10 ABSTRACT

11 Symbiotic microalgae (Symbiodinium spp.) strongly influence the performance and stress-

12 tolerance of their coral hosts, making the analysis of Symbiodinium communities in corals

13 (and metacommunities on reefs) advantageous for many aspects of coral reef research.

14 High-throughput sequencing of ITS2 nrDNA offers unprecedented scale in describing these

15 communities, yet high intragenomic variability at this locus complicates the resolution of

16 biologically meaningful diversity. Here, we demonstrate that generating operational

17 taxonomic units by clustering ITS2 sequences at 97\% similarity within, but not across,

18 samples collapses sequence diversity that is more likely to be intragenomic, while

19 preserving diversity that is more likely interspecific. We utilize this 'within-sample

20 clustering' to analyze Symbiodinium from ten host taxa on shallow reefs on the north and

21 south shores of St. John, US Virgin Islands. While Symbiodinium communities did not differ

22 between shores, metacommunity network analysis of host-symbiont associations revealed

23 Symbiodinium lineages occupying 'dominant' and 'background' niches, and coral hosts that 
24 are more 'flexible' or 'specific' in their associations with Symbiodinium. These methods

25 shed new light on important questions in coral symbiosis ecology, and demonstrate how

26 application-specific bioinformatic pipelines can improve the analysis of metabarcoding

27 data in microbial metacommunity studies.

\section{INTRODUCTION}

29 The composition of symbiotic algal communities (Symbiodinium spp.) in reef-building 30 scleractinian and milleporine corals plays a major role in their biology and ecology, as

31 identity and the functional performance of symbionts influences emergent properties of

32 the holobiont, including its photobiology, energetics, growth rates, and susceptibility to

33 stress (Rowan, 2004; Cantin et al., 2009; Jones and Berkelmans, 2011). Even slight

34 differences in the relative abundance of different algal symbionts may have important

35 functional consequences for the host (Loram et al., 2007; Cunning et al., 2015a; Bay et al.

36 2016). Moreover, variation in these communities among individuals within a host species,

37 and within individuals over time (Thornhill et al. 2006; Edmunds et al. 2014), is an

38 important trait modulating sensitivity of corals to environmental stress (Putnam et al.,

39 2012), and their ability to respond in beneficial ways to changing environmental conditions

40 by 'shuffling' or 'switching' symbionts (Baker, 2003). Therefore, an accurate and

41 comprehensive description of Symbiodinium communities within coral hosts informs

42 understanding of the ecological performance of corals.

43 Symbiodinium identity currently is primarily described using genetic sequences of

44 chloroplast (cp23S and psbA ${ }^{\text {ncr }}$ ) and nuclear markers (18S, 28S, ITS1, and ITS2) 
45 (LaJeunesse, 2001; Santos et al., 2003; Pochon et al., 2012). Together, these markers have 46 been used to identify nine major 'clades' within the genus Symbiodinium (clades A through

47 I (Pochon and Gates, 2010)), which have been further divided into 'types' based on the 48 marker with which they are identified.. Symbiodinium species are beginning to be described based on combinations of markers, including microsatellites to establish reproductive isolation (i.e., to satisfy the biological species concept) (LaJeunesse et al., 2014; Thornhill et al. 2014). However, ecological surveys of Symbiodinium diversity still generally rely on commonly-used marker genes, such as ITS2. Consequently, high-throughput sequencing of

53 ITS2 is being utilized to characterize Symbiodinium communities with unprecedented 54 resolution (e.g., Kenkel et al. 2013; Arif et al., 2014; Green et al. 2014; Quigley et al., 2014, 2016; Thomas et al., 2014; Edmunds et al., 2014; Cunning et al., 2015b; Ziegler et al., 2017). However, such analyses often create datasets consisting of millions of sequence reads and

57 hundreds of thousands of distinct sequence variants (Ziegler et al., 2017), which places great importance on the ways in which ITS2 sequence diversity is analyzed and interpreted

59 in relation to biological diversity.

While ITS2 initially was investigated as a potential species-level marker in Symbiodinium (LaJeunesse, 2001), it is now understood that this marker is not sufficiently variable to distinguish all species within this genus (Finney et al., 2010; Thornhill et al., 2013). For example, the 'B1' ITS2 sequence variant is shared by S. minutum, S. pseudominutum, and $S$. antillogorgium, and potentially other species (Parkinson et al., 2015). Moreover, the position of ITS2 within the tandemly-repeating ribosomal DNA array creates multiple ITS2 sequence variants within a single genome (Thornhill et al., 2007) that evolve through concerted evolution (e.g. (Dover, 1986)). In fact, concerted evolution may mask species 
68 divergence within Symbiodinium by maintaining ancestral sequence variants as numerical

69 dominants in multiple derived lineages (Thornhill et al., 2013). Together, these features of

70 ITS2 complicate the interpretation of intragenomic versus interspecific variation, and

71 preclude its use as a true species-level marker for Symbiodinium. Nevertheless, numerically

72 dominant intragenomic variants of ITS2 are still phylogenetically informative across the

73 genus (LaJeunesse, 2001), and resolve diversity at a functionally and ecologically important

74 level. Moreover, due to the large quantity of existing sequence data for comparative

75 analysis (e.g., (Franklin et al., 2012; Tonk et al., 2013)), and the relative ease of amplifying

76 and sequencing ITS2, it remains an essential and powerful marker for Symbiodinium.

77 Therefore, it is important to develop best practices in the bioinformatic analysis and

78 interpretation of ITS2 metabarcoding surveys of Symbiodinium.

79 In addressing this objective, we test the ability of 'within-sample clustering' (i.e.

80 independently clustering sequences at $97 \%$ similarity within each sample) to generate

81 biologically relevant operational taxonomic units (OTUs) from ITS2 metabarcoding data.

82 Specifically, this approach addresses the fact that dominant ITS2 sequences from different

83 Symbiodinium species may be more similar to one another than intragenomic variants

84 within one Symbiodinium (Thornhill et al., 2013; Arif et al., 2014; Parkinson et al., 2015).

85 Therefore, clustering all sequences together may underestimate diversity by collapsing

86 different species into the same OTU. Conversely, treating each ITS2 sequence as a unique

87 Symbiodinium type may overestimate diversity due to intragenomic variation (Thornhill et

88 al. 2007). Within-sample clustering may better approximate true diversity by exploiting

89 key assumptions regarding the distribution of symbionts among samples, and sequences

90 among symbionts. These assumptions include: 1) that most coral colonies are dominated 
91 by a single Symbiodinium type (LaJeunesse and Thornhill, 2011; Pettay et al., 2011; Baums

92 et al., 2014; but see Silverstein et al. 2012), and 2) that different numerically dominant ITS2

93 sequence variants identify different Symbiodinium types, even when they differ by only 1

94 nucleotide (Sampayo et al., 2009). These assumptions suggest that when closely related

95 ITS2 sequences (i.e., that are >97\% similar; Arif et al. (2014)) occur within the same

96 sample, they are more likely to be intragenomic variants, but when they are numerically

97 dominant in different samples, they are more likely to represent distinct Symbiodinium

98 taxa. Accordingly, clustering sequences at 97\% similarity within each sample

99 independently may collapse variability that is more likely to be intragenomic (i.e.,

100 occurring within a sample), while maintaining variability that is more likely to be

101 interspecific (i.e., occurring in different samples).

102 We demonstrate this within-sample clustering approach with an analysis of Symbiodinium

103 communities in ten host species across the north and south shores of St. John. We explore

104 ecological patterns that can be revealed by large-scale metabarcoding datasets, including 1)

105 testing for whole-community differences associated with north and south shore locations,

106 2) analyzing coral-Symbiodinium metacommunity association networks, and 3) quantifying

107 the variability in symbiont communities (i.e., beta diversity, or 'symbiosis flexibility')

108 among individuals within a host species. The large quantity of data, and the kinds of

109 analytical approaches facilitated by ITS2 metabarcoding, has the potential to revolutionize

110 understanding of Symbiodinium metacommunity ecology in reef corals and other taxa

111 harboring similar symbionts. More generally, the present study demonstrates how

112 ecological knowledge can inform bioinformatic analyses using marker genes for which

113 sequence diversity does not map directly to species. 


\section{MATERIALS AND METHODS}

\section{Sample collection and environmental conditions}

116 Eighty-four tissue samples were collected from ten species of scleractinian and milleporine

117 corals at seven sites around St. John, US Virgin Islands, USA (Fig. 1), between August 7th

118 and 9th, 2012, as permitted by the Virgin Islands National Park (permit VIIS-2012-SCI-

119 0017). Hosts were sampled at sites on both the north and south shores of St. John (except

120 for 0 . annularis and $P$. astreoides that were only sampled on the south shore). Samples were

121 collected on snorkel from 3-7m depth, and colonies to be sampled were selected

122 haphazardly in upward-facing locations exposed to ambient irradiance. Tissue biopsies

123 ( $\sim 5$-mm diameter and $\sim 5$-mm depth) were removed from coral colonies with metal

124 borers, and the holes were plugged with non-toxic modeling clay.

125 Broad-scale differences in the hydrographic regimes at points representative of the north

126 and south shores of St. John (North: $18.368141^{\circ} \mathrm{N}, 64.711967^{\circ} \mathrm{W}$; South: $18.315465^{\circ} \mathrm{N}$,

$12764.726840^{\circ} \mathrm{W}$ ) were evaluated from remote sensing tools to determine sea surface

128 temperature $\left({ }^{\circ} \mathrm{C}\right)$, surface chlorophyll concentrations ( $\left.\mathrm{mg} \mathrm{chl} \mathrm{a} \mathrm{m}^{-3}\right)$, and integrated wave

129 exposure on a power scale $\left(\mathrm{J} \mathrm{m}^{-3}\right)$. These features were selected as they corresponded to

130 anecdotal observations of differences between the water masses on both shores, and

131 conditions known to affect coral performance, Symbiodinium biology (i.e., SST (Coles and

132 Jokiel, 1977) and wave regime (Atkinson et al., 1994)), and the supply of food resources

133 that play important roles in coral nutrition (Houlbrèque and Ferrier-Pagès, 2009). SST and

134 chlorophyll were determined using data from the MODIS sensor on the Aqua satellite. SST

135 was evaluated from nighttime records, and chlorophyll from ocean colour, both of which 
136 were obtained at $1 \mathrm{~km}$ spatial resolution for each month, averaged over the years 2003-

137 2010, from the IMaRS website (http://imars.usf.edu/modis); if data were not available at

138 the chosen coordinates (above), values from the nearest available pixel were used.

139 Boxplots were generated from the 12 monthly climatological values obtained for each

140 response (Fig. 1).

141 Wind-driven wave exposure on a power scale for a given site is dependent on the wind

142 patterns and the configuration of the coastline, which defines the fetch. To calculate wave

143 exposure, wind speed and direction at each location were acquired from the QuickSCAT

144 (NASA) satellite scatterometer from 1999 to 2008 at $25 \mathrm{~km}$ spatial resolution (Hoffman

145 and Leidner, 2005). Coastline data were obtained from the Global Self-consistent,

146 Hierarchical, High-resolution, Shoreline (GSHHS v 2.2) database, which provides global

147 coastline at 1:250,000 scale (Wessel and Smith, 1996). From these data, wave exposure

148 was calculated using the methods based on wave theory (after Chollett et al. 2012) for 32

149 fetch directions (equally distributed through $360^{\circ}$ ). Total wave exposure (summed over all

150 directions) was calculated in R using the packages maptools (Bivand and Lewin-Koh,

151 2016), raster (Hijmans, 2016), rgeos (Bivand and Rundel, 2016), and sp (Bivand et al., 152 2013).

153 ITS2 sequencing and bioinformatics

154 Coral tissue samples were preserved in $\sim 500 \mu \mathrm{L}$ Guanidinium buffer $(50 \% \mathrm{w} / \mathrm{v}$ 155 guanidinium isothiocyanate; 50 mM Tris pH 7.6; $10 \mu \mathrm{M}$ EDTA; 4.2\% w/v sarkosyl; 2.1\%

$156 \mathrm{v} / \mathrm{v} \beta$-mercaptoethanol) and shipped to the Hawaii Institute of Marine Biology (HIMB).

157 Genomic DNA was extracted from each coral tissue sample following a guanidinium-based 
158 extraction protocol (Cunning et al., 2015b), and sent to Research and Testing Laboratory

159 (Lubbock, TX) for sequencing of ITS2 amplicons ('itsD' and 'its2rev2' primers from Stat et

160 al. (2009)) on the Illumina MiSeq platform with 2x300 paired-end read chemistry.

161 Paired reads from each sample (provided in fastq format by Research and Testing

162 Laboratory) were merged using illumina-utils software (Eren et al., 2013b) with an

163 enforced Q30-check and an overlap $\geq 150$ bp with $\leq 3$ mismatches required to retain a

164 sequence. Chimeric sequences were removed using usearch61 (Edgar, 2010) implemented

165 in QIIME (Caporaso et al., 2010). Primers were trimmed using cutadapt (Martin, 2011)

166 allowing 3 mismatches, and only sequences with both forward and reverse primer matches

167 and length $\geq 250 \mathrm{bp}$ after trimming were retained. Subsequently, three different

168 clustering approaches, each based on the uclust algorithm (Edgar, 2010) and implemented

169 in QIIME, were used to group ITS2 sequences into operational taxonomic units (OTUs): 1)

170 clustering at $100 \%$ identity, 2) clustering at $97 \%$ identity across samples (i.e., sequences

171 from all samples clustered together), and 3) clustering at 97\% identity within samples (i.e.,

172 sequences from each sample clustered independently). For each $97 \%$ cluster, the most

173 abundant sequence variant was chosen as the representative sequence, and within-sample

174 clusters were merged across samples if their representative sequences were $100 \%$

175 identical. After removing singleton clusters, representative sequences for each OTU were

176 assigned taxonomy by searching a custom reference database of Symbiodinium ITS2

177 sequences using the Needleman-Wunsch global alignment algorithm implemented in the

178 Biostrings package (Pagès et al., 2016) in R (R Core Team, 2014). Each OTU was then

179 assigned a name corresponding to the reference sequence(s) with the highest alignment

180 score; if the match was $<100 \%$, the assignment was given a unique superscript. If the match 
181 was $<90 \%$, the sequence was blasted to the NCBI nt database and omitted if the top hit did

182 not contain "Symbiodinium".

183 The reference database comprised Symbiodinium ITS2 sequences downloaded directly

184 from NCBI. These sequences included those used in previous reference databases (Cunning

185 et al., 2015b) supplemented with additional sequences of Symbiodinium from Caribbean

186 corals (Finney et al., 2010; Green et al., 2014). Reference sequences were separated and

187 aligned by clade using muscle (Edgar, 2004), and trimmed to equal length using the o-

188 smart-trim command from oligotyping software (Eren et al., 2013a), before being

189 reconcatenated into a single fasta file. The bioinformatic pipeline used here was

190 implemented using a series of bash scripts, which can be found in the data archive

191 accompanying this paper (http://github.com/jrcunning/STJ2012). These scripts are

192 provided along with all raw data and a Makefile, which can be executed to fully reproduce

193 the present analysis.

194 Symbiodinium data analysis

195 OTU tables, sample metadata, and taxonomic data were imported into R using the phyloseq 196 package (McMurdie and Holmes, 2013) to facilitate downstream analyses. OTU tables were 197 filtered to remove any OTU that was not observed at least 10 times, or any sample with < 198200 sequences (to remove possible sequencing errors and samples that did not sequence 199 well), and counts were transformed to relative abundance. Permutational analysis of 200 variance (PERMANOVA) was used to test for differences in Symbiodinium community 201 composition between the north and south shores of St. John within each coral species. 
202 Network analysis and visualization was performed in R using the igraph package (Csárdi

203 and Nepusz, 2006). Networks were created featuring 'dominant' ( $>50 \%$ relative

204 abundance) and 'abundant' (>1\% relative abundance) OTUs, with weighted edges

205 proportional to the number of individuals within a species in which a symbiont OTU

206 occurred. A 'background' symbionts network was also created with unweighted edges

207 defined based on the presence of a symbiont at $<1 \%$ relative abundance in at least one

208 individual within the species. To simplify visualization of the background symbiont

209 network, clade D OTUs were merged into a single node under the assumption that clade D

210 in the Caribbean comprises a single species (Symbiodinium trenchii; (LaJeunesse et al.,

2112014 )), and symbiont nodes connected to $\leq 2$ coral species were removed from the

212 network. All network layouts were constructed based on the Fruchterman-Reingold

213 algorithm (Fruchterman and Reingold, 1991).

214 Beta diversity (sensu (Anderson et al., 2006)) was calculated as the multivariate dispersion

215 of samples within a coral species. Principal coordinate analysis of Bray-Curtis

216 dissimilarities of square-root transformed OTU counts was used to calculate average

217 distance-to-centroid values for each species, which were then compared statistically by a

218 permutation test. This analysis was implemented using the betadisper function in the

219 vegan package (Oksanen et al., 2016), based on (Anderson et al., 2006). 


\section{RESULTS}

\section{Comparison of clustering approaches}

222 The 84 coral samples of ten host species generated 1,490,813 sequences after merging

223 paired reads, removing chimeric sequences, and trimming primers. After clustering, OTUs

224 with $<10$ sequences and samples with $<200$ sequences were excluded, leaving 80 samples

225 for downstream analysis. The number of OTUs resolved, as well as the number of

226 sequences per OTU and per sample, depended on the clustering approach (Table 1). More

227 OTUs were resolved as the clustering resolution increased (i.e., 97\% OTUs across samples <

228 97\% OTUs within samples < 100\% OTUs), with fewer reads per OTU (Table 1). The number

229 of sequences per sample was less for the 100\% OTU approach because more OTUs were

230 filtered out of the dataset by the minimum threshold count of 10 reads per OTU.

231 A subset of samples was selected for comparative analysis of the community structure

232 resolved by the three clustering approaches (Fig. 2). Despite being dominated by different

233 sequence variants (Fig. 2A), across-sample clustering assigned the same dominant OTU to

234 many of these samples (Fig. 2B), whereas within-sample clustering assigned them different

235 OTUs (Fig. 2C) corresponding to their dominant sequence variants. Overall, across-sample

236 clustering collapsed more sequence diversity into a single OTUs, while within-sample

237 clustering resolved more distinct OTUs that associated with particular host species.

238 Because the outcomes of the latter approach are more consistent with the current

239 understanding of ITS2 sequence diversity as it links to Symbiodinium biology and ecology

240 (see Discussion), the remainder of the results is presented using the $97 \%$ within-sample

241 clustering approach. Detailed analysis and visualizations comparing the outcomes of the 
242 three clustering approaches for all individuals of each coral species are available at

243 http://github.com/jrcunning/STJ2012.

\section{Symbiodinium community composition}

245 Within the set of coral samples, clade B had the highest relative abundance (46.6\%),

246 followed by clade C (41.3\%), A (10.5\%), D (1.5\%), and G (0.1\%). The number of OTUs

247 within each clade followed a similar pattern, with the highest number in clade B (66),

248 followed by clade C (25), A (6), D (5), and G (4). The distribution of these clades within each

249 sample is shown in Fig. 3.

\section{Differences in Symbiodinium between shores}

251 The environmental conditions broadly characterizing the north and south shores are

252 presented in Fig. 1. From 2003 to 2010, the north shore of St. John was characterized by

253 slightly lower sea surface temperatures, higher chlorophyll a concentrations, and lower

254 wave exposure, relative to the south shore. No significant differences in Symbiodinium

255 community composition between the north and south shores were detected for any host

256 species (Table 2). However, qualitative differences were apparent: Porites furcata was

257 dominated by either clade A or clade C Symbiodinium on the south shore, but only by clade

$258 \mathrm{C}$ on the north shore (difference between shores PERMANOVA: $\mathrm{p}=0.056$ ). Siderastrea

259 siderea was dominated by either clade C or clade D on the south shore, but only by clade C

260 on the north shore (difference between shores PERMANOVA: $\mathrm{p}=0.071$ ). 


\section{Network analysis of Symbiodinium metacommunity}

262 Patterns of association between hosts and Symbiodinium were analyzed using networks

263 focusing on "abundant" (>1\% relative abundance; Fig. 4), "dominant" (>50\% relative

264 abundance; Fig. 5A), and "background" ( $<1 \%$ relative abundance; Fig. 5B) symbionts in

265 each host species, based on 97\% within-sample clustering. (Networks for individuals

266 within each species are available at http://github.com/jrcunning/STJ2012). In the network

267 for abundant symbionts (Fig. 4), 37 OTUs were observed. P. strigosa associated with the

268 greatest number of OTUs (n=17), followed by F. fragum $(\mathrm{n}=5), M$. alcicornis $(\mathrm{n}=5)$, and $D$.

269 cylindrus $(\mathrm{n}=4)$; these primarily comprised OTUs closely related to B1 and B19. In contrast,

270 only a single OTU occurred at $>1 \%$ relative abundance in both $P$. astreoides (A4) and $M$.

271 cavernosa (C3). All coral species hosted at least one "abundant" Symbiodinium OTU that

272 was also abundant in at least one other coral species, except for S. radians. This species was

273 dominated by C46, but also contained Symbiodinium closely related to B1 and B19, as well

274 as B5.

275 In the network for dominant symbionts, 15 different Symbiodinium OTUs were included,

276 defined as those occurring at $>50 \%$ relative abundance within a host (Fig. 5A). A single

277 OTU identical to Symbiodinium B1 was the most prevalent dominant symbiont, dominating

278 all D. cylindrus samples and many P. strigosa (4 of 9), O. annularis (2 of 4), M. alcicornis (6 of

279 10), and $F$. fragum (8 of 9) samples. Other closely-related clade B OTUs (similar to B1, B1d,

280 and B19) occasionally dominated P. strigosa, M. alcicornis, and F. fragum, while O. annularis

281 was dominated just as often by Symbiodinium C7. After B1, the next most prevalent

282 dominant symbionts were Symbiodinium C3, which dominated all M. cavernosa and most $S$. 
283 siderea (7 of 10), and Symbiodinium A4, which dominated all P. astreoides and 3 of $10 P$.

284 furcata. P. furcata and S. siderea were each occasionally dominated by other symbiont

285 OTUs, including C45 or C45a (P. furcata), and C1 or D1 (S. siderea). All coral species were

286 dominated by at least one Symbiodinium OTU that also dominated at least one other coral

287 species, except for S. radians, which was exclusively dominated by C46.

288 There were 6 symbionts that occupied a 'background' niche, defined as $<1 \%$ relative

289 abundance in three or more coral species (Fig. 5B). A member of Symbiodinium clade D

290 (which has been described as S. trenchii (LaJeunesse et al., 2014)) was found at background

291 levels in the greatest number of host species (7), followed by A4 and C3 (both in 6 taxa).

292 C31, C46, and B1 were each detected at background levels in 4 host species.

\section{Symbiotic flexibility of host species}

294 Symbiont communities from each individual host were ordinated in multivariate space, and

295 the dispersion of individuals within each host species (i.e., average distance to centroid)

296 was calculated as a metric of symbiosis 'flexibility'. Higher values indicate hosts with

297 greater variability in symbiont community composition among individuals, and lower

298 values indicating hosts with greater uniformity. This metric does not reflect how many

299 Symbiodinium OTUs are hosted by a given coral species, but rather how much variation in

300 Symbiodinium community composition occurs among different individuals. This metric

301 revealed significant differences in symbiotic flexibility among host species (Fig. 6). The

302 highest distance to centroid was found in P. strigosa, P. furcata, M. alcicornis, S. siderea, and

303 M. annularis, and the lowest was found in M. cavernosa. Flexibility was also low in $D$.

304 cylindrus, S. radians, and P. astreoides, and intermediate in F. fragum. 


\section{DISCUSSION}

\section{Bioinformatic analysis of ITS2 diversity}

307 The structure of Symbiodinium communities that was detected in host species was

308 impacted by the bioinformatic approach used to analyze ITS2 sequence data. Comparing 309 taxonomic assignments of the OTUs generated by these approaches demonstrates that

310 across-sample clustering often assigns the same dominant OTU to samples with different

311 dominant sequence variants (Fig. 2), while within-sample clustering assigns them distinct

312 OTUs that reflect the dominant variant in the sample. This occurs because OTU identity is

313 determined by the most abundant member sequence, and when members occur across

314 many samples (i.e., during across-sample clustering), each member's abundance reflects

315 the number (and sequencing depth) of samples in the dataset that contain it. Therefore, a

316 particular sequence variant from a more extensively-sampled and/or deeply-sequenced

317 host may determine the identity of an OTU that includes closely-related but different

318 sequences in other samples, even though they do not actually contain the given sequence.

319 Such merging of OTUs across samples is undesirable, since even single nucleotide

320 differences in a sample's dominant ITS2 variant may indicate different Symbiodinium

321 species (Sampayo et al., 2009).

322 Therefore, even though clustering at 97\% similarity may be needed to collapse

323 intragenomic variation within a single Symbiodinium (Arif et al., 2014), when such

324 clustering is applied across a dataset potentially comprised of many closely related

325 Symbiodinium, it is likely to also collapse interspecific variation and underestimate 326 diversity. This limitation was also encountered by Arif et al. (2014) when clustering 
327 closely-related clade C sequences across samples from multiple hosts. Despite being

328 comprised mostly of C41 variants, 3,000 ITS2 sequences from Acropora hemprichii were

329 subsumed into a single OTU that was named C1 after the dominant variant among the

$330 \sim 7,000$ sequences from Pocillopora verrucosa with which they were clustered. In this case

331 (and as shown in Fig. 2), clustering sequences across samples leads to the conclusion that

332 samples with different dominant sequence variants are dominated by the same

333 Symbiodinium OTU. This outcome occurs because the identity of OTUs in any given sample

334 may be determined by other samples in the dataset; indeed, using this approach, the same

335 sample and sequence assemblage may receive different OTU assignments if it were part of

336 a different set of samples.

337 Meanwhile, clustering sequences within each sample independently assigns OTUs that

338 reflect only the assemblage of sequences within that sample, and therefore does not

339 depend on the presence or abundance of sequences from other samples included in the

340 analysis. The outcome of within-sample clustering of ITS2 sequences as applied herein

341 better reflects patterns of Symbiodinium diversity and ecology that have been revealed by

342 more variable markers (psbA ${ }^{\text {ncr }}$ ) and microsatellites (Finney et al., 2010; Thornhill et al.,

343 2013); namely, that clades B and $C$ in the Caribbean comprise numerous different

344 Symbiodinium species that tend to associate with different coral host taxa. Indeed, only

345 within-sample clustering assigned different dominant clade C OTUs to samples of $O$.

346 annularis and S. siderea (Fig. 2), which have been differentiated based on other markers

347 (Thornhill et al., 2013). The within-sample clustering approach should also reflect patterns

348 that would be observed by sequencing dominant ITS2 bands from denaturing gradient gel

349 electrophoresis (DGGE; the most commonly use method of describing Symbioinium 
350 diversity prior to metabarcoding), since both methods rely on numerically dominant

351 sequence variants to assign taxonomy. Furthermore, metabarcoding overcomes the

352 primary limitations of the DGGE method by providing more quantitative data and sensitive

353 detection of low abundance taxa (Quigley et al., 2014). Therefore, we recommend a within-

354 sample clustering approach for metabarcoding studies where many different Symbiodinium

355 types are likely to be encountered, but suggest that additional work with Symbiodinium

356 cultures would help further validate this method.

357 Importantly, the assumptions underlying a within-sample clustering approach -- that

358 samples typically contain one Symbiodinium species that can be diagnosed by its most

359 abundant intragenomic ITS2 variant -- will not always be met. Indeed, multiple

360 Symbiodinium frequently co-occur in single coral colonies (Silverstein et al., 2012), which

361 undermines support for assuming that variation within a sample is intragenomic. However,

362 co-occurring Symbiodinium in many cases are members of different clades, whose ITS2

363 sequences are divergent enough to be resolved separately by $97 \%$ clustering. Thus, only

364 when very closely related types co-occur in a sample (e.g., Sampayo et al., 2007; Wham and

365 LaJeunesse, 2016) is this approach likely to fail. Other problematic cases may occur when

366 multiple intragenomic sequence variants are co-dominant (i.e., comprise nearly equal

367 proportions of the rDNA array), such that slight differences in their relative abundance may

368 lead to different OTU assignments in samples with otherwise highly similar sequence

369 assemblages. These challenges may be overcome by basing taxonomic assignment on more

370 complex criteria than just the most abundant sequence variant, such as multiple co-

371 dominant variants, or more evolutionarily derived variants. Alternatively, other markers 
372 (mitochondrial, microsatellites and flanking regions) may help overcome these taxonomic

373 challenges (Stat et al. 2012).

374 While no treatment of ITS2 data can provide species-level descriptions of Symbiodinium

375 communities (since the marker itself does not provide this resolution), within-sample

376 clustering nevertheless accommodates known evolutionary complexities in the ITS2 locus

377 to provide community descriptions that better reflect species-level diversity than either

378 across-sample clustering or no clustering at all. When treated with an appropriate

379 bioinformatic approach, ITS2 metabarcoding can provide a comprehensive and

380 quantitative analysis of Symbiodinium ITS2 diversity, and can be applied across host

381 species to rapidly survey the Symbiodinium metacommunity on a reef-wide scale. To

382 complement this field-based study, we suggest that sequencing of prescribed mixtures of

383 cultured Symbiodinium species could strengthen support for this approach, and identify

384 cases in which it could be improved.

\section{Symbiodinium metacommunity ecology in St. John}

386 In the ten host species sampled, the most prevalent Symbiodinium were members of clades

387 B and C, which is consistent with previous analyses of Symbiodinium diversity on shallow

388 reefs in this region (LaJeunesse, 2004; Correa et al., 2009; Finney et al., 2010; Edmunds et

389 al. 2014). Less frequent associations between hosts and members of clades A and D were

390 observed, although clade A dominated P. astreoides and some P. furcata, and clade D

391 dominated one $S$. siderea. Finally, clade G was observed at low relative abundances $(<5 \%)$

392 in some coral colonies, which likely would not have been detected without high-throughput

393 sequencing. 
394 While the environmental conditions differed between the north and south shores of St.

395 John (e.g., wave exposure, temperature, and chlorophyll a), there were no statistically

396 significant differences in symbiont community composition in the corals sampled on either

397 shore. This may partly be due to small sample sizes, since two species showed a trend for

398 differences between shores: S. siderea and P. furcata were more frequently dominated by

399 Symbiodinium in clades D and A (respectively) on the south shore, compared to the north

400 shore (Fig. 2). Symbiodinium in clades D and A are typically associated with warm and

401 variable temperatures (e.g., Baker et al., 2004; Oliver and Palumbi, 2011) and shallow

402 habitats with high light intensities (LaJeunesse, 2002). Therefore, it is consistent with

403 expectations that they were more prevalent on the south shore, where temperatures were

404 slightly higher, and chlorophyll levels were lower, suggesting greater light penetration into

405 the water column. However, the lack of differentiation between shores in the symbiont

406 communities of most host species suggests that environmental differences at this relatively

407 small scale are not major drivers of Symbiodinium community structure.

408 Network analysis of associations between Symbiodinium types and coral species revealed

409 metacommunity-level patterns in Symbiodinium ecology. First, ITS2 type B1 was

410 "abundant" (i.e. >1\% relative abundance in a sample) in seven of ten host species (Fig. 4),

411 and "dominant" (i.e. >50\%) in five of ten (Fig. 5), suggesting it is a generalist symbiont in St.

412 John. However, analysis of other markers such as chloroplast and microsatellite loci has

413 revealed that Symbiodinium ITS2 type B1 in the Caribbean is comprised of multiple lineages

414 that show high host species fidelity (Santos et al., 2004; Finney et al., 2010; Parkinson et al.,

415 2015). Likewise, the apparent generalists Symbiodinium C3 (which dominated colonies of $S$.

416 siderea and M. cavernosa) and Symbiodinium A4 (which dominated P. astreoides and $P$. 
417 furcata, Fig. 5) may also be comprised of multiple host-specialized lineages (Thornhill et al., 418 2013), which could be revealed using higher resolution genetic markers.

419 In addition to hosting apparent generalist ITS2 lineages, most coral species sampled in St.

420 John (except M. cavernosa and P. astreoides) hosted abundant Symbiodinium OTUs that

421 were not abundant in any other corals, and are thus apparently more host-specific. Unique

422 OTUs in the B1-radiation were abundant in M. alcicornis and D. cylindrus, while others from

423 both the B1- and B19-radiations (see LaJeunesse, 2004) were abundant in F. fragum, $S$.

424 radians, and $P$. strigosa. Several clade C OTUs similarly were only abundant in one species:

425 C1144 in P. strigosa, C7 in O. annularis, C1 in S. siderea, C1c/C45 and C45a in P. furcata, and

426 C46 in S. radians. Interestingly, S. radians was the only species in which none of the

427 abundant symbionts occurred in any other host, which may reflect the unique ecology of $S$.

428 radians as the only study species that typically forms small, encrusting colonies as adults.

429 To reveal symbionts that consistently occupied a 'background' niche, we identified OTUs

430 present at $<1 \%$ relative abundance in samples of three or more host species. This

431 distribution suggests that these symbionts can occupy a range of hosts, but are unlikely to

432 be dominant symbionts, at least under prevailing environmental conditions. While it is

433 possible that some of these may be free-living surface contaminants not in symbiosis with

434 the host (sensu Silverstein et al. 2012), the most prevalent 'background' symbiont in St.

435 John was a member of clade D (Symbiodinium trenchii (LaJeunesse et al., 2014)), which is

436 known to proliferate within hosts during and after thermal stress (Thornhill et al., 2006;

437 LaJeunesse et al., 2009; Silverstein et al., 2015), or in marginal environments (LaJeunesse et 438 al., 2010). Other symbionts occupying a background niche in St. John, such as Symbiodinium 
439 A4 and C3, may similarly become dominant under different sets of environmental

440 conditions. These background symbionts, therefore, may perform an important functional

441 role within the metacommunity by broadening the fundamental niche that a host may

442 occupy (sensu Bruno et al. (2003)), thereby increasing its resilience to environmental

443 change (Correa and Baker, 2011).

444 Indeed, the ability of corals to associate with different symbionts may be an important trait

445 that mediates their sensitivity to stress (Putnam et al., 2012), and their ability to change

446 symbionts over time in response to environmental change (Baker, 2003). While flexibility

447 in symbiosis ecology has been quantified previously for individual corals (i.e., based on the

448 diversity of symbionts co-occurring within a single host colony (Putnam et al., 2012)), here

449 we quantify flexibility at the host species level (Fabina et al. 2012, 2013) based on

450 quantitative variability of symbiont community structure among multiple colonies (Fig. 6),

451 a metric of beta diversity that can be statistically compared among host species (Anderson

452 et al., 2006). This metric revealed that M. cavernosa, P. astreoides, $S$. radians, and $D$.

453 cylindrus had the lowest symbiotic flexibility, meaning that all sampled individuals had

454 similar symbiont community structure. In these host species, symbiont communities may

455 be more constrained by host biology and/or less responsive to environmental variation. On

456 the other hand, P. strigosa, P. furcata, M. alcicornis, and S. siderea had high flexibility,

457 meaning that sampled individuals displayed more divergent symbiont communities.

458 Community structure in these hosts may be more responsive to variability in the

459 environment (e.g., Kennedy et al., 2016), or subject to greater stochasticity. While

460 comparisons among studies are often confounded by methodology and location, the

461 flexibility of these species in St. John is generally congruent with broad-scale patterns of 
462 symbiont associations recorded in the Coral Traits database (coraltraits.org; Madin et al.

463 2012). While future work should investigate whether the scope for symbiont community

464 change over time within individuals is linked to variability among individuals, we suggest

465 that the latter represents a useful metric of symbiosis flexibility that can be easily

466 quantified using metabarcoding data.

\section{Conclusions}

468 ITS2 metabarcoding and within-sample OTU clustering represents a powerful approach to 469 quantitatively and comprehensively describe Symbiodinium metacommunity composition 470 on coral reefs. The scale and resolution of datasets generated in this way facilitate new

471 analytical applications that can address critical topics in coral symbiosis ecology, including

472 changes in dominant and background symbionts across environmental gradients and over

473 time, and the role of metacommunity processes in shaping Symbiodinium communities.

474 Describing these trends has the potential to greatly advance understanding of coral 475 responses to environmental change.

\section{Acknowledgements}

477 Remote sensing observations of sea surface temperature and phytoplankton pigment

478 concentration were processed at the Institute for Marine Remote Sensing at the College of

479 Marine Science, University of South Florida, in St. Petersburg, Florida, and provided

480 courtesy of Frank Müller-Karger and Iliana Chollett. 


\section{References}

482 Anderson MJ, Ellingsen KE, McArdle BH. (2006). Multivariate dispersion as a measure of 483 beta diversity. Ecology Letters 9: 683-693.

484 Arif C, Daniels C, Bayer T, Banguera-Hinestroza E, Barbrook AC, Howe CJ, LaJeunesse TC, 485 Voolstra CR. (2014). Assessing Symbiodinium diversity in scleractinian corals via next486 generation sequencing-based genotyping of the ITS2 rDNA region. Molecular Ecology 23: $487 \quad 4418-4433$.

488 Atkinson MJ, Kotler E, Newton P. (1994). Effects of water velocity on respiration, 489 calcification, and ammonium uptake of a Porites compressa community. Pacific Science 48: $490 \quad 296-303$.

491 Baker AC. (2003). Flexibility and specificity in coral-algal symbiosis: diversity, ecology, and 492 biogeography of Symbiodinium. Annual Review of Ecology, Evolution, and Systematics 34: 493 661-689.

494 Baker AC, Starger C, McClanahan TR, Glynn PW. (2004). Corals' adaptive response to 495 climate change. Nature 430: 741.

496 Bay LK, Doyle J, Logan M, Berkelmans R. (2016). Recovery from bleaching is mediated 497 by threshold densities of background thermo-tolerant symbiont types in a reef-building 498 coral. Royal Society Open Science 3: 160322.

499 Baums IB, Devlin-Durante MK, LaJeunesse TC. (2014). New insights into the dynamics 500 between reef corals and their associated dinoflagellate endosymbionts from population 501 genetic studies. Molecular Ecology 23: 4203-4215. 
502 Bivand R, Lewin-Koh N. (2016). maptools: Tools for reading and handling spatial objects.

503 Bivand R, Pebesma E, Gomez-Rubio V. (2013). Applied spatial data analysis with R. 2nd ed. 504 Springer: New York.

505 Bivand R, Rundel C. (2016). rgeos: Interface to Geometry Engine - Open Source (GEOS).

506 Bruno JF, Stachowicz J, Bertness M. (2003). Inclusion of facilitation into ecological theory.

507 Trends in Ecology and Evolution 18: 119-125.

508 Cantin NE, van Oppen MJH, Willis BL, Mieog JC, Negri AP. (2009). Juvenile corals can

509 acquire more carbon from high-performance algal symbionts. Coral Reefs 28: 405-414.

510 Caporaso JG, Kuczynski J, Stombaugh JI, Bittinger K, Bushman FD, Costello EK, Fierer N,

511 Gonzalez Pena A, Goodrich JK, Gordon JI, Huttley GA, Kelley ST, Knights D, Koenig JE, Ley

512 RE, Lozupone CA, McDonald D, Muegge BD, Pirrung M, Reeder J, Sevinsky JR, Turnbaugh PJ, 513 Walters WA, Widmann J, Yatsunenko T, Zaneveld J, Knight R. (2010). QIIME allows analysis

514 of high-throughput community sequencing data. Nature Methods 7: 335-336.

515 Chollett I, Mumby PJ (2012). Predicting the distribution of Montastraea reefs using wave 516 exposure. Coral Reefs 31: 493-503.

517 Coles S, Jokiel PL. (1977). Effects of temperature on photosynthesis and respiration in 518 hermatypic corals. Marine Biology 43: 209-216.

519 Correa AMS, Baker AC. (2011). Disaster taxa in microbially mediated metazoans: how 520 endosymbionts and environmental catastrophes influence the adaptive capacity of reef 521 corals. Global Change Biology 17: 68-75. 
522 Correa AMS, Brandt ME, Smith TB, Thornhill DJ, Baker AC. (2009). Symbiodinium

523 associations with diseased and healthy scleractinian corals. Coral Reefs 28: 437-448.

524 Csárdi G, Nepusz T. (2006). The igraph software package for complex network research.

525 InterJournal, Complex Systems. http://igraph.com.

526 Cunning R, Silverstein RN, Baker AC. (2015a). Investigating the causes and consequences of 527 symbiont shuffling in a multi-partner reef coral symbiosis under environmental change.

528 Proceedings of the Royal Society B 282: 20141725.

529 Cunning R, Yost DM, Guarinello ML, Putnam HM, Gates RD. (2015b). Variability of

530 Symbiodinium communities in waters, sediments, and corals of thermally distinct reef pools

531 in American Samoa. PLoS ONE 10: e0145099.

532 Dover G. (1986). Molecular drive in multigene families: How biological novelties arise,

533 spread and are assimilated. Trends in Genetics 2: 159-165.

534 Edgar RC. (2004). MUSCLE: multiple sequence alignment with high accuracy and high 535 throughput. Nucleic acids research 32: 1792-1797.

536 Edgar RC. (2010). Search and clustering orders of magnitude faster than BLAST.

537 Bioinformatics 26: 2460-2461.

538 Edmunds PJ, Pochon X, Levitan DR, Yost DM, Belcaid M, Putnam HM, Gates RD. (2014).

539 Long-term changes in Symbiodinium communities in Orbicella annularis in St. John, US

540 Virgin Islands. Marine Ecology Progress Series 506: 129-144. 
541 Eren AM, Maignien L, Sul WJ, Murphy LG, Grim SL, Morrison HG, Sogin ML. (2013a).

542 Oligotyping: differentiating between closely related microbial taxa using 16S rRNA gene 543 data. Methods in Ecology and Evolution 4: 1111-1119.

544 Eren AM, Vineis JH, Morrison HG, Sogin ML. (2013b). A filtering method to generate high

545 quality short reads using Illumina paired-end technology. PLOS ONE 8: e66643.

546 Fabina NS, Putnam HM, Franklin EC, Stat M, Gates RD. (2012). Transmission mode predicts

547 specificity and interaction patterns in coral-Symbiodinium networks. PLoS ONE, 7(9), $548 \mathrm{e} 44970$.

549

550 Fabina NS, Putnam HM, Franklin EC, Stat M, Gates RD. (2013). Symbiotic specificity, 551 association patterns, and function determine community responses to global changes: 552 defining critical research areas for coral-Symbiodinium symbioses. Global Change Biology 553 19: 3306-3316.

554 Finney J, Sampayo EM, Warner ME, Oxenford H, LaJeunesse TC. (2010). The relative 555 significance of host-habitat, depth, and geography on the ecology, endemism, and 556 speciation of coral endosymbionts in the genus Symbiodinium. Microbial Ecology 60: 250557263.

558 Franklin EC, Stat M, Pochon X, Putnam HM, Gates RD. (2012). GeoSymbio: a hybrid, cloud559 based web application of global geospatial bioinformatics and ecoinformatics for 560 Symbiodinium-host symbioses. Molecular Ecology Resources 12: 369-373.

561 Fruchterman TMJ, Reingold EM. (1991). Graph drawing by force-directed placement. 562 Software: Practice and Experience 21: 1129-1164. 
563 Green EA, Davies SW, Matz MV, Medina M. (2014). Quantifying cryptic Symbiodinium

564 diversity within Orbicella faveolata and Orbicella franksi at the Flower Garden Banks, Gulf 565 of Mexico. PeerJ 2: e386.

566 Hijmans RJ. (2016). raster: Geographic Data Analysis and Modeling.

567 Hoffman R, Leidner SM. (2005). An introduction to the near-real-time QuikSCAT data.

568 Weather and Forecasting 20: 476-493.

569 Houlbrèque F, Ferrier-Pagès C. (2009). Heterotrophy in tropical scleractinian corals.

570 Biological Reviews 84: 1-17.

571 Jones AM, Berkelmans R. (2011). Tradeoffs to thermal acclimation: Energetics and

572 reproduction of a reef coral with heat tolerant Symbiodinium type-D. Journal of Marine

573 Biology 2011: 185890.

574 Kennedy EV, Tonk L, Foster NL, Chollett I, Ortiz JC, Dove SG, Hoegh-Guldberg HG, Mumby

575 PJ, Stevens JR. (2016). Symbiodinium biogeography tracks environmental patterns rather

576 than host genetics in a key Caribbean reef-builder, Orbicella annularis. Proceedings of the

577 Royal Society B 283: 20161938-918.

578 Kenkel CD, Goodbody-Gringley G, Caillaud D, Davies SW, Bartels E, Matz MV. (2013).

579 Evidence for a host role in thermotolerance divergence between populations of the

580 mustard hill coral (Porites astreoides) from different reef environments. Molecular Ecology

$581 \quad 22: 4335-4348$.

582 LaJeunesse TC. (2002). Diversity and community structure of symbiotic dinoflagellates

583 from Caribbean coral reefs. Marine Biology 141: 387-400. 
584 LaJeunesse TC. (2001). Investigating the biodiversity, ecology, and phylogeny of

585 endosymbiotic dinoflagellates in the genus Symbiodinium using the ITS region: in search of

586 a 'species' level marker. Journal of Phycology 37: 866=880.

587 LaJeunesse TC. (2005). 'Species' radiations of symbiotic dinoflagellates in the Atlantic and

588 Indo-Pacific since the Miocene-Pliocene transition. Molecular Biology And Evolution 22:

$589 \quad 570-581$.

590 LaJeunesse TC, Pettay DT, Sampayo EM, Phongsuwan N, Brown BE, Obura DO, Hoegh-

591 Guldberg HG, Fitt WK (2010). Long-standing environmental conditions, geographic

592 isolation and host-symbiont specificity influence the relative ecological dominance and

593 genetic diversification of coral endosymbionts in the genus Symbiodinium. Journal of

594 Biogeography 37: 785-800.

595 LaJeunesse TC, Smith RT, Finney J, Oxenford HA. (2009). Outbreak and persistence of

596 opportunistic symbiotic dinoflagellates during the 2005 Caribbean mass coral 'bleaching'

597 event. Proceedings of the Royal Society B 276: 4139-4148.

598 LaJeunesse TC, Thornhill DJ. (2011). Improved resolution of reef-coral endosymbiont

599 (Symbiodinium) species diversity, ecology, and evolution through psbA non-coding region

600 genotyping. PLOS ONE 6: e29013.

601 LaJeunesse TC, Wham DC, Pettay DT, Parkinson JE, Keshavmurthy S, Chen CA. (2014).

602 Ecologically differentiated stress-tolerant endosymbionts in the dinoflagellate genus

603 Symbiodinium (Dinophyceae) Clade D are different species. Phycologia 53: 305-319. 
604 Loram JE, Trapido-Rosenthal HG, Douglas AE. (2007). Functional significance of genetically

605 different symbiotic algae Symbiodinium in a coral reef symbiosis. Molecular Ecology 16:

$606 \quad 4849-4857$.

607 Madin JS, Hoogenboom MO, Connolly SR, Darling ES, Falster DS, Huang D, Keith SA, Mizerek

608 T, Pandolfi JM, Putnam HM, Baird AH. (2016). A trait-based approach to advance coral reef

609 science. Trends in Ecology and Evolution 31: 419-428.

610 Martin M. (2011). Cutadapt removes adapter sequences from high-throughput sequencing

611 reads. EMBnetjournal 17: pp. 10-12.

612 McMurdie PJ, Holmes S. (2013). phyloseq: an R package for reproducible interactive

613 analysis and graphics of microbiome census data. PLOS ONE 8: e61217.

614 Oksanen J, Blanchet FG, Kindt R, Legendre P, Minchin PR, OHara RB, Simpson GL, Solymos

615 P, Stevens MHH, Soecs E, Wagner H. (2016). vegan: Community Ecology Package.

616 http://CRAN.R-project.org/package=vegan.

617 Oliver TA, Palumbi SR. (2011). Do fluctuating temperature environments elevate coral

618 thermal tolerance? Coral Reefs 30: 429-440.

619 Pagès H, Aboyoun P, Gentleman R, DebRoy S. (2016). Biostrings: String objects

620 representing biological sequences, and matching algorithms.

621 Parkinson JE, Coffroth MA, LaJeunesse TC. (2015). New species of clade B Symbiodinium

622 (Dinophyceae) from the greater Caribbean belong to different functional guilds: $S$.

623 aenigmaticum sp. nov., S. antillogorgium sp. nov., S. endomadracis sp. nov., and $S$.

624 pseudominutum sp. nov. Journal of Phycology 51: 850-858. 
625 Pettay DT, Wham DC, Pinzón JH, LaJeunesse TC. (2011). Genotypic diversity and

626 spatialtemporal distribution of Symbiodinium clones in an abundant reef coral. Molecular

627 Ecology 20: 5197-5212.

628 Pochon X, Gates RD. (2010). A new Symbiodinium clade (Dinophyceae) from soritid

629 foraminifera in Hawai'i. Molecular Phylogenetics and Evolution 56: 492-497.

630 Pochon X, Putnam HM, Burki F, Gates RD. (2012). Identifying and characterizing alternative

631 molecular markers for the symbiotic and free-living dinoflagellate genus Symbiodinium.

632 PLOS ONE 7: e29816.

633 Putnam HM, Stat M, Pochon X, Gates RD. (2012). Endosymbiotic flexibility associates with

634 environmental sensitivity in scleractinian corals. Proceedings of the Royal Society B 279:

$635 \quad 226-235$.

636 Quigley KM, Davies SW, Kenkel CD, Willis BL, Matz MV, Bay LK. (2014). Deep-sequencing

637 method for quantifying background abundances of Symbiodinium types: exploring the rare

638 Symbiodinium biosphere in reef-building corals. PLoS ONE 9: e94297.

639 Quigley KM, Willis BL, Bay LK. (2016). Maternal effects and Symbiodinium community

640 composition drive differential patterns in juvenile survival in the coral Acropora tenuis.

641 Royal Society Open Science 3: 160471.

642 R Core Team. (2014). R: A language and environment for statistical computing.

643 http://www.R-project.org/.

644 Rowan R. (2004). Coral bleaching: thermal adaptation in reef coral symbionts. Nature 430: 645742. 
646 Sampayo EM, Dove SG, LaJeunesse TC. (2009). Cohesive molecular genetic data delineate

647 species diversity in the dinoflagellate genus Symbiodinium. Molecular Ecology 18: 500-519.

648 Sampayo EM, Franceschinis L, Hoegh-Guldberg O, Dove SG. (2007). Niche partitioning of

649 closely related symbiotic dinoflagellates. Molecular Ecology 16: 3721-3733.

650 Santos SR, Gutierrez-Rodriguez C, Coffroth MA. (2003). Phylogenetic identification of

651 symbiotic dinoflagellates via length heteroplasmy in domain $\mathrm{V}$ of chloroplast large subunit

652 (cp23S) ribosomal DNA sequences. Marine Biotechnology 5: 130-140.

653 Santos SR, Shearer TL, Hannes AR, Coffroth MA. (2004). Fine-scale diversity and specificity

654 in the most prevalent lineage of symbiotic dinoflagellates (Symbiodinium, Dinophyceae) of

655 the Caribbean. Molecular Ecology 13: 459-469.

656 Silverstein RN, Correa AMS, Baker AC. (2012). Specificity is rarely absolute in coral-algal

657 symbiosis: implications for coral response to climate change. Proceedings of the Royal

658 Society B 239: 2609-2618.

659 Silverstein RN, Cunning R, Baker AC. (2015). Change in algal symbiont communities after

660 bleaching, not prior heat exposure, increases heat tolerance of reef corals. Global Change

661 Biology 21: 236-249.

662 Stat M, Baker AC, Bourne DG, Correa AMS, Forsman Z, Huggett MJ, Pochon X, Skillings D,

663 Toonen RJ, van Oppen MJH, Gates RD. (2012). Molecular delineation of species in the coral

664 holobiont. Advances in Marine Biology 63: 1-65.

665 Stat M, Pochon X, Cowie ROM, Gates RD. (2009). Specificity in communities of

666 Symbiodinium in corals from Johnston Atoll. Marine Ecology Progress Series 386: 83-96. 
667 Thomas L, Kendrick GA, Kennington WJ, Richards ZT, Stat M. (2014). Exploring

668 Symbiodinium diversity and host specificity in Acropora corals from geographical extremes 669 of Western Australia with 454 amplicon pyrosequencing. Molecular Ecology 23: 31136703126.

671 Thornhill DJ, LaJeunesse TC, Kemp DW, Fitt WK, Schmidt GW. (2006). Multi-year, seasonal 672 genotypic surveys of coral-algal symbioses reveal prevalent stability or post-bleaching 673 reversion. Marine Biology 148: 711-722.

674 Thornhill DJ, LaJeunesse TC, Santos SR. (2007). Measuring rDNA diversity in eukaryotic 675 microbial systems: how intragenomic variation, pseudogenes, and PCR artifacts confound 676 biodiversity estimates. Molecular Ecology 16: 5326-5340.

677 Thornhill DJ, Lewis AM, Wham DC, LaJeunesse TC. (2014). Host-specialist lineages

678 dominate the adaptive radiation of reef coral endosymbionts. Evolution 68: 352-367.

679 Tonk L, Bongaerts P, Sampayo EM, Hoegh-Guldberg O. (2013). SymbioGBR: a web-based 680 database of Symbiodinium associated with cnidarian hosts onthe Great Barrier Reef. BMC 681 Ecology 13: 1-1.

682 Wessel P, Smith WHF. (1996). A global, self-consistent, hierarchical, high-resolution 683 shoreline database. Journal of Geophysical Research B: Solid Earth 101: 8741-8743.

684 Wham DC, LaJeunesse TC. (2016). Symbiodinium population genetics: testing for species 685 boundaries and analysing samples with mixed genotypes. Molecular Ecology 25: 26996862712. 
687 Ziegler M, Arif C, Burt JA, Dobretsov S, Roder C, LaJeunesse TC, Voolstra CR. (2017).

688 Biogeography and molecular diversity of coral symbionts in the genus Symbiodinium

689 around the Arabian Peninsula. Journal of Biogeography. e-pub ahead of print, doi:

$690 \quad 10.1111 /$ jbi.12913. 


\section{Figure and Table Legends}

693 Figure 1. Sampling of Symbiodinium communities from ten host species (a) on the north

694 and south shores of St. John, US Virgin Islands, and the broad-scale physical conditions

695 associated with these two environments (b-d). Mapped points along each shore indicate

696 locations from which biological samples were collected, while ' $N$ ' and 'S' indicate locations

697 from which environmental data representative of the north and south shores were

698 obtained. Insets present boxplots of sea-surface temperatures (b) and chlorophyll a

699 concentrations (c), and a barplot of the sum total wave exposure (d) recorded at these two

700 locations.

701

702

703

704

705

706

707

708

709

710

711

712

713

714

715

716

717

718

719

720

721

722

723

724

725

726

727

728

729

730

731

732

Figure 2. Comparison of dominant OTUs assigned by $100 \%, 97 \%$ across-sample, and $97 \%$ within-sample clustering approaches. A) The composition of unique sequence variants (i.e., $100 \%$ OTUs) in each sample, sorted by relative abundance, and filled by unique colors corresponding to unique sequence variants. Sequence variants are annotated with the name of their best-matching reference sequence, with brackets indicating the match is not exact (only OTUs with a relative abundance $>0.05$ are annotated for clarity). The dominant OTUs within each sample assigned by B) $97 \%$ across-sample clustering and C) $97 \%$ withinsample clustering are shown by rectangles below each bar, with fill colors matching the unique sequence variants presented in (A) to indicate the representative sequence of the assigned OTU. Colored rectangles that span multiple bars indicate that the corresponding samples were assigned the same dominant OTU.

Figure 3. Symbiodinium community composition of each sample. Samples are plotted as horizontal bars, sorted by species and shore of collection (north vs. south). Individual segments of each bar represent $97 \%$ within-sample OTUs, colored by Symbiodinium clade identity.

Figure 4. Network analysis of abundant Symbiodinium taxa in each coral species. Symbiodinium OTUs (circular nodes) are connected to each coral species (square nodes) in which they ever occurred at $>1 \%$ relative abundance within an individual; thickness of edges (i.e., links between coral and symbiont nodes) is relative to the proportion of individuals within the coral species in which the Symbiodinium OTU was present at $>1 \%$. Symbiont nodes are colored by clade identity, and sized proportionally to the number of coral species in which they were found.

Figure 5. Network analysis of dominant (A) and background (B) Symbiodinium taxa in each coral species (see Materials and Methods for details on network construction). Symbiont nodes are colored by clade identity, sized proportionally to the number of coral species to which they are connected.

Figure 6. Symbiotic flexibility in coral species quantified as multivariate dispersion of Symbiodinium community composition (mean distance to centroid) \pm SE. Host taxa that do not share a letter are significantly different $(\mathrm{p}<0.05)$.

Table 1. Summary statistics for each clustering approach.

Table 2. Mean overall, within-shore ('within'), and between-shore ('between') Bray-Curtis dissimilarities of the Symbiodinium communities in each host species, and PERMANOVA 
733 tests (partial $\mathrm{R}^{2}$ and p-values) for a difference between shores. Between-shore tests were 734 not possible for $O$. annularis and P. astreoides since they were only sampled from one shore. 
Figure 1

Sampling locations and environmental conditions

Sampling of Symbiodinium communities from ten host species (a) on the north and south shores of St. John, US Virgin Islands, and the broad-scale physical conditions associated with these two environments (b-d). Mapped points along each shore indicate locations from which biological samples were collected, while ' $N$ ' and ' $S$ ' indicate locations from which environmental data representative of the north and south shores were obtained. Insets present boxplots of sea-surface temperatures (b) and chlorophyll a concentrations (c), and a barplot of the sum total wave exposure (d) recorded at these two locations.

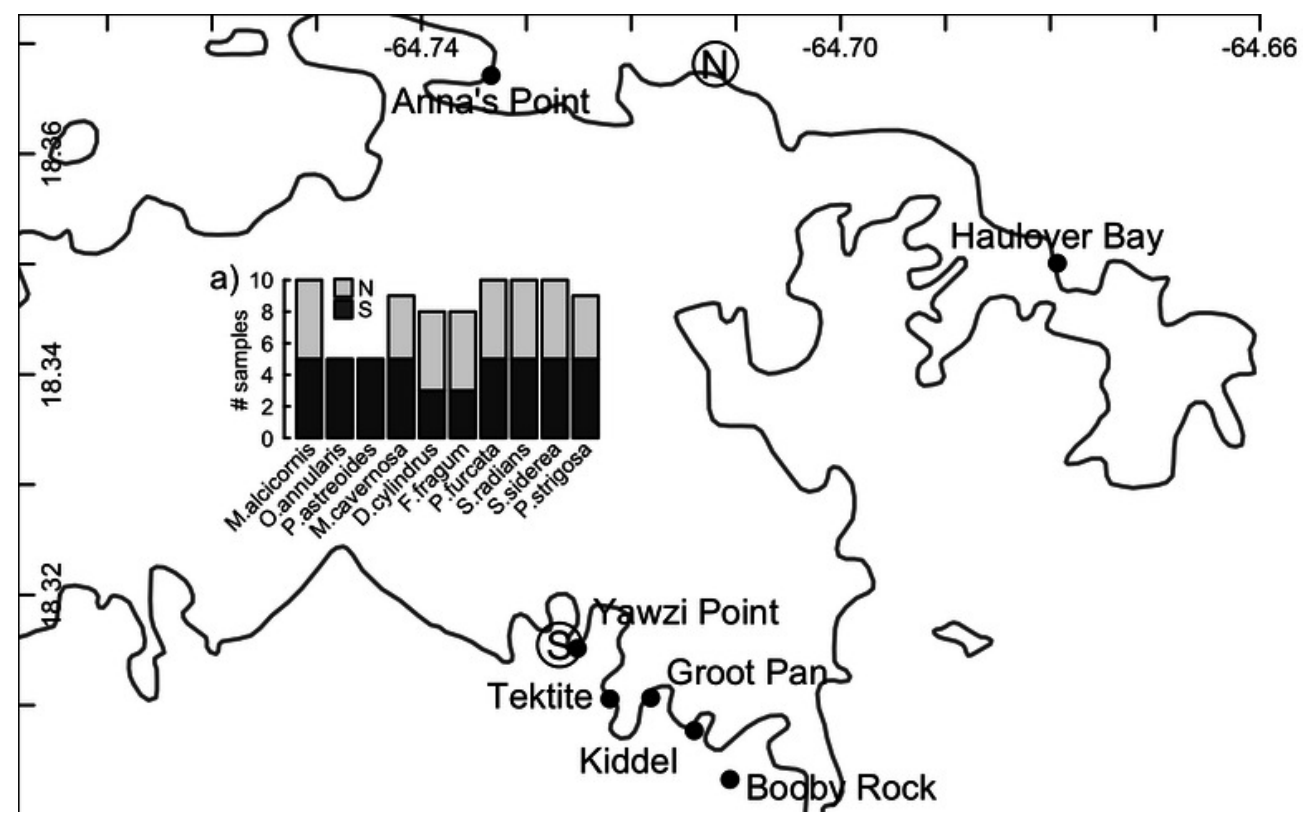

b)

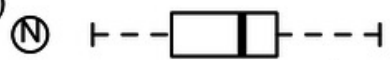

(S)

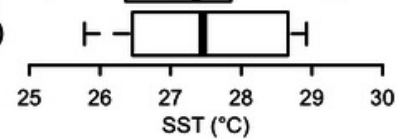

c)

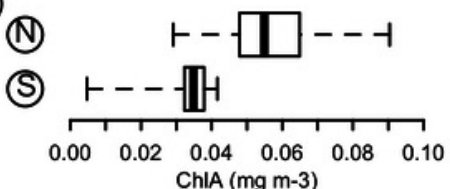

d)

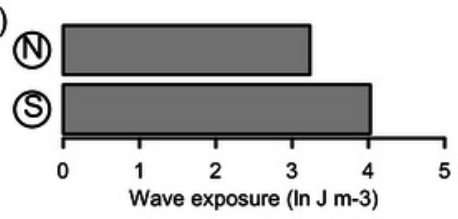




\section{Figure 2}

OTU assignments by different clustering approaches

Comparison of dominant OTUs assigned by 100\%, $97 \%$ across-sample, and $97 \%$ withinsample clustering approaches. A) The composition of unique sequence variants (i.e., 100\% OTUs) in each sample, sorted by relative abundance, and filled by unique colors corresponding to unique sequence variants. Sequence variants are annotated with the name of their best-matching reference sequence, with brackets indicating the match is not exact (only OTUs with a relative abundance $>0.05$ are annotated for clarity). The dominant OTUs within each sample assigned by B) $97 \%$ across-sample clustering and C) $97 \%$ within-sample clustering are shown by rectangles below each bar, with fill colors matching the unique sequence variants presented in $(A)$ to indicate the representative sequence of the assigned OTU. Colored rectangles that span multiple bars indicate that the corresponding samples were assigned the same dominant OTU. 
A.

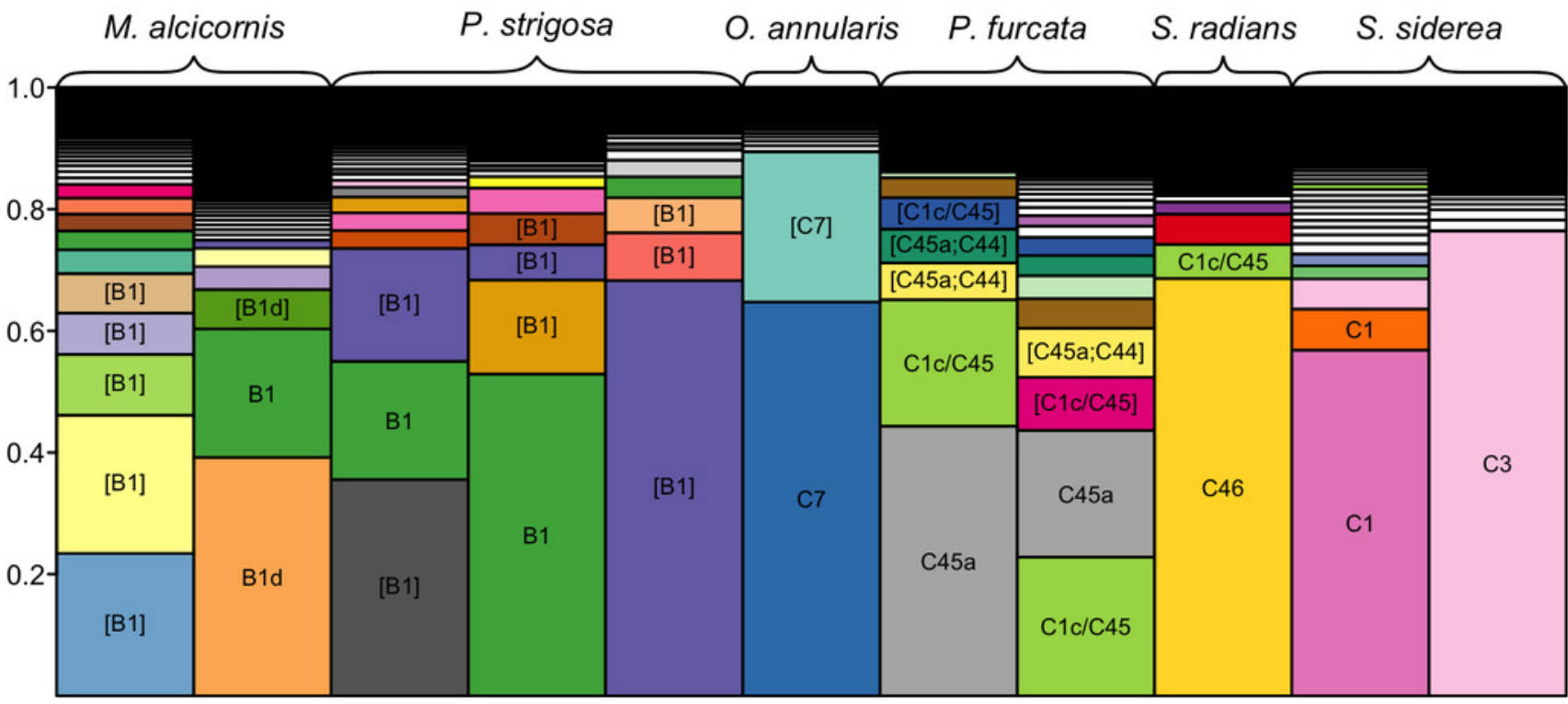

B.

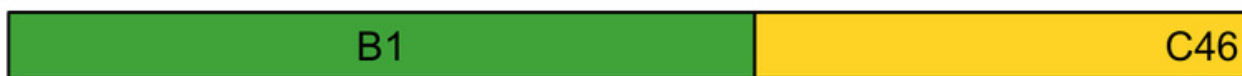

C.

\begin{tabular}{|l|l|l|l|l|l|l|l|l|l|l|}
\hline [B1] & B1d & {$[\mathrm{B} 1]$} & $\mathrm{B} 1$ & {$[\mathrm{~B} 1]$} & $\mathrm{C} 7$ & $\mathrm{C} 45 \mathrm{a}$ & $\mathrm{C} 1 \mathrm{C} / \mathrm{C} 45$ & $\mathrm{C} 46$ & $\mathrm{C} 1$ & $\mathrm{C} 3$ \\
\hline
\end{tabular}




\section{Figure 3}

Symbiodinium community composition of each sample

Samples are plotted as horizontal bars, sorted by species and shore of collection (north vs. south). Individual segments of each bar represent $97 \%$ within-sample OTUs, colored by Symbiodinium clade identity. 


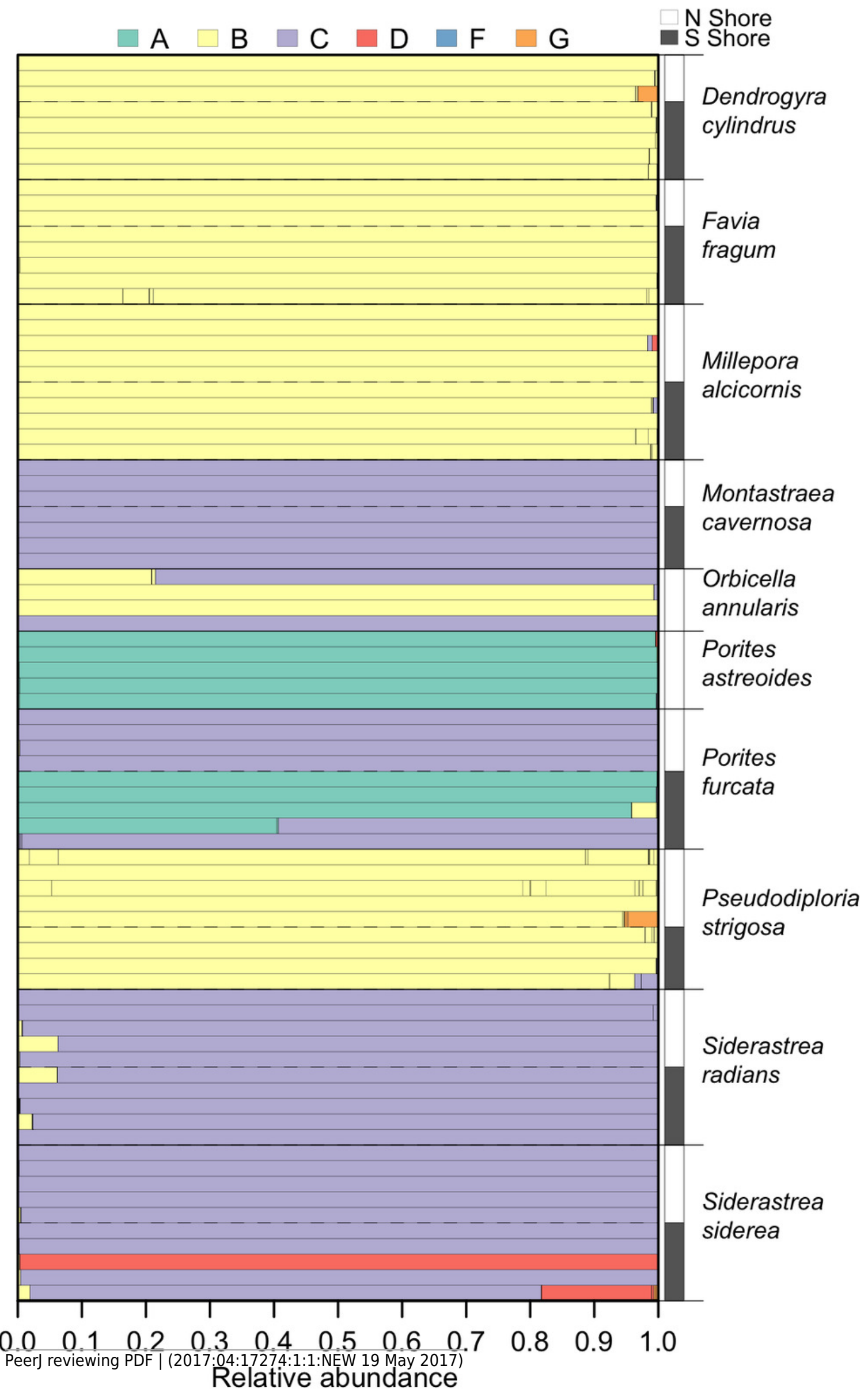




\section{Figure 4}

Network analysis of abundant Symbiodinium taxa in each coral species.

Symbiodinium OTUs (circular nodes) are connected to each coral species (square nodes) in which they ever occurred at $>1 \%$ relative abundance within an individual; thickness of edges (i.e., links between coral and symbiont nodes) is relative to the proportion of individuals within the coral species in which the Symbiodinium OTU was present at $>1 \%$. Symbiont nodes are colored by clade identity, and sized proportionally to the number of coral species in which they were found. 


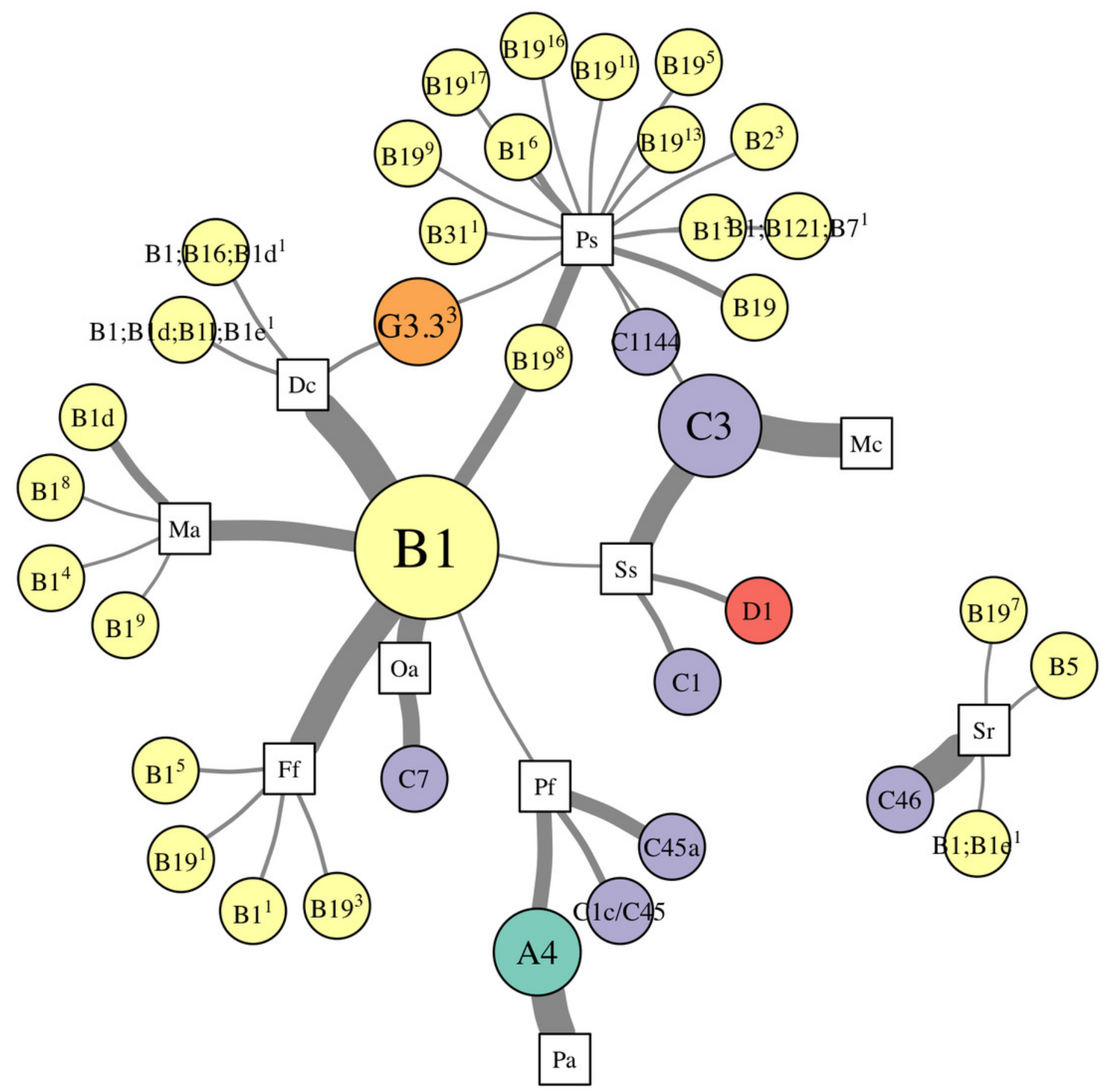


Figure 5

Network analysis of dominant and background symbionts

Network analysis of dominant (A) and background (B) Symbiodinium taxa in each coral species (see Materials and Methods for details on network construction). Symbiont nodes are colored by clade identity, sized proportionally to the number of coral species to which they are connected.

A.)

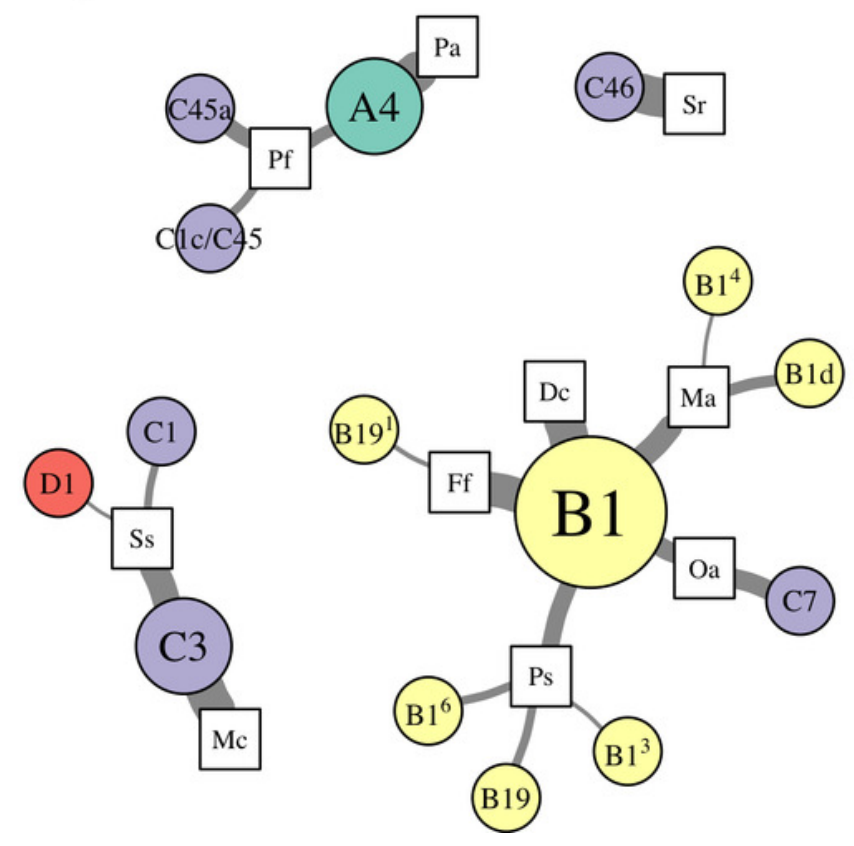

B.)

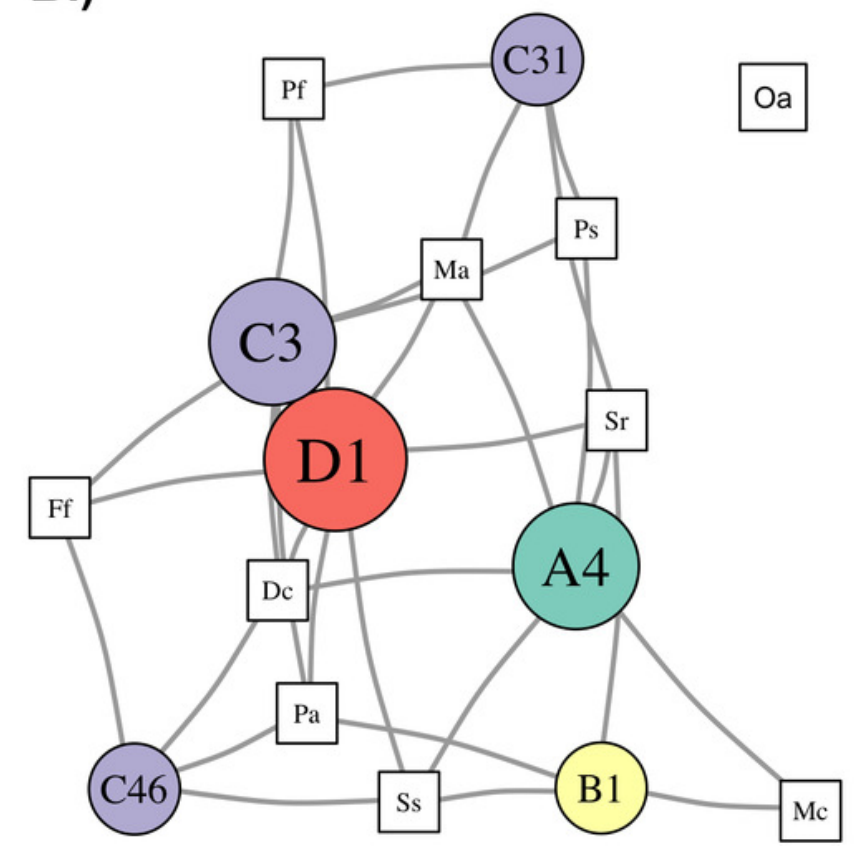


Figure 6

Symbiotic flexibility (beta diversity) of host species.

Symbiotic flexibility in coral species quantified as multivariate dispersion of Symbiodinium community composition (mean distance to centroid) \pm SE. Host taxa that do not share a letter are significantly different $(p<0.05)$.

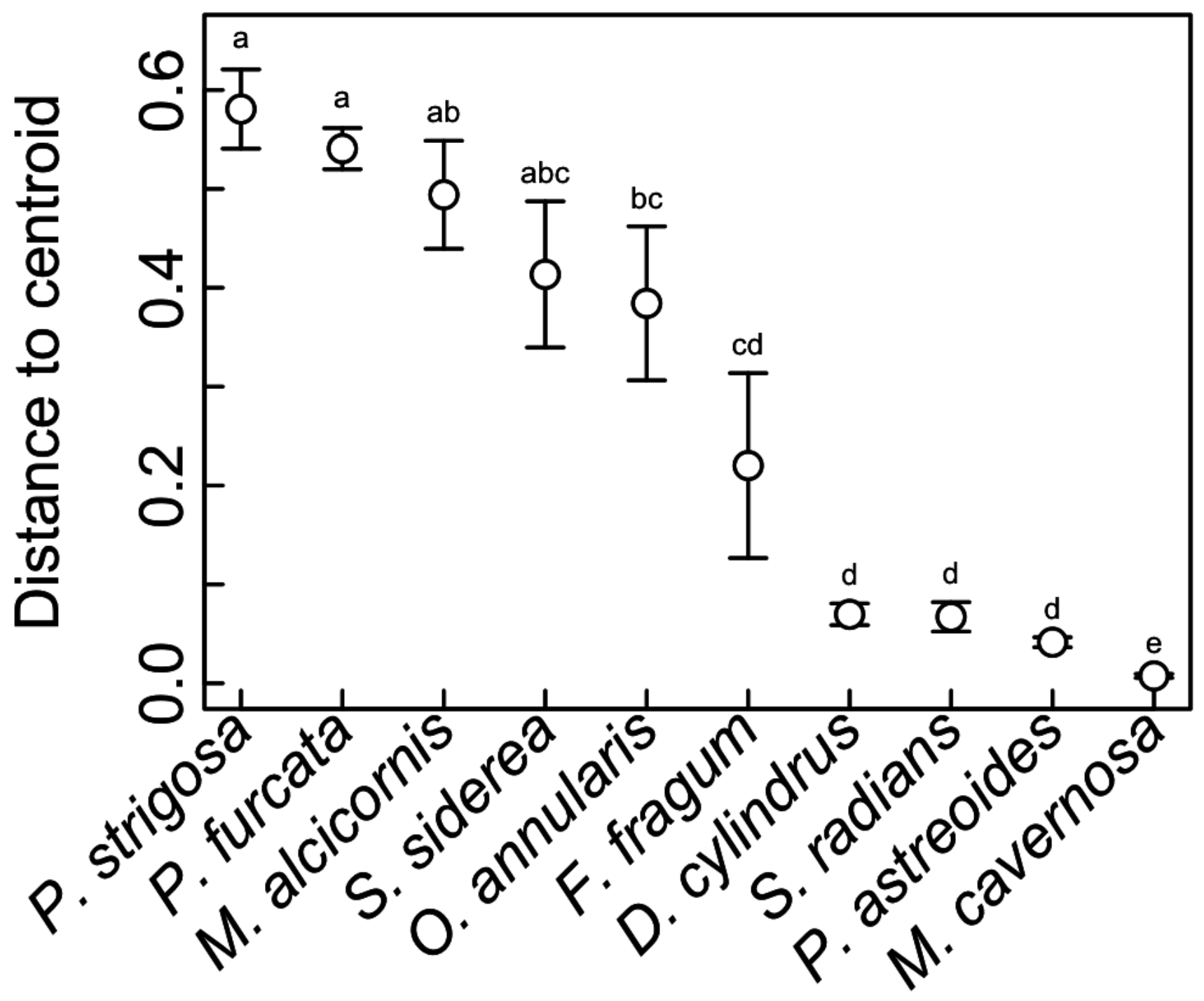




\section{Table 1 (on next page)}

Summary statistics for each clustering approach. 
97\% OTUs

across samples

1

Number of OTUs

Range of OTU counts

94

$10-742671$

$706-169884$

$13141 * / 2$

Geometric mean (*/ GSD)

reads per sample

1
97\% OTUs

within samples

106

$10-472752$

$707-169890$

$13137 * / 2$

$100 \%$ OTUs

4718

$10-171212$

$485-97003$

$8141 * / 2$ 


\section{Table 2 (on next page)}

Differences in Symbiodinium communities within and between shores

Mean overall, within-shore ('within'), and between-shore ('between') Bray-Curtis dissimilarities of the Symbiodinium communities in each host species, and PERMANOVA tests (partial $\mathrm{R}^{2}$ and $\mathrm{p}$-values) for a difference between shores. Between-shore tests were not possible for $O$. annularis and $P$. astreoides since they were only sampled from one shore. 
Millepora alcicornis

\begin{tabular}{rrrrrr}
$\mathrm{n}$ & overall & within & between & $R^{2}$ & $p$ \\
\hline 10 & 0.604 & 0.653 & 0.564 & 0.037 & 0.809 \\
4 & 0.632 & 0.632 & - & - & - \\
5 & 0.004 & 0.004 & - & - & - \\
7 & 0 & 0.001 & 0 & 0.118 & 1 \\
8 & 0.017 & 0.016 & 0.019 & 0.188 & 0.268 \\
8 & 0.251 & 0.309 & 0.202 & 0.086 & 0.75 \\
9 & 0.703 & 0.555 & 0.821 & 0.345 & 0.056 \\
10 & 0.03 & 0.031 & 0.029 & 0.064 & 0.953 \\
10 & 0.512 & 0.541 & 0.489 & 0.061 & 0.467 \\
9 & 0.789 & 0.71 & 0.851 & 0.242 & 0.079
\end{tabular}

